\title{
Normative dimensions and symmetry of the lacrimal drainage system on dacryocystography: statistical analysis of morphometric characteristics
}

\author{
A. Horsburgh1, T.F. Massoud 1, 2,3 \\ 'Section of Neuroradiology and Head and Neck Imaging, Department of Radiology, Addenbrooke's Hospital, \\ United Kingdom \\ 2University of Cambridge, Cambridge, United Kingdom \\ ${ }^{3}$ Department of Radiology, Stanford University School of Medicine, USA
}

[Received 28 November 2012; Accepted 14 December 2012]

\begin{abstract}
Background: Asymmetric lacrimal flow occurs in females more than males. We hypothesised that the normal lacrimal drainage system (LDS) may show subtle left-right asymmetry in morphometry when imaged on dacryocystography (DCG).

Materials and methods: We retrospectively reviewed 60 normal bilateral LDSs. Images were analysed for 7 parameters: (1) combined length of either the superior or inferior canaliculus plus common canaliculus, (2) angle of insertion of common canaliculus into lacrimal sac $(L S),(3,4)$ length and width of the $L S,(5)$ length of the nasolacrimal duct (NLD), and $(6,7)$ width of proximal and distal NLD. We tested the effect of independent variables (age, gender, and side) on each dependent variable (length, width, or angle) using regression analysis. We used a Student t-test for independent samples to statistically compare bilateral LDSs.

Results: Patient median age was 62 years, and male:female ratio was 27:73. Mean dimensions and angles for all LDSs were: (1) $14.5 \mathrm{~mm}$, (2) $57^{\circ}$, (3) $11.6 \mathrm{~mm}$, (4) $2.1 \mathrm{~mm}$, (5) $20.7 \mathrm{~mm}$, (6) $1.4 \mathrm{~mm}$, and (7) $1.8 \mathrm{~mm}$. No independent variable affected variation in length, width, or angle. No significant difference emerged between dimensions of right and left LDSs in both sexes. Conclusions: A degree of affective lateralisation in the brain is known to result in lacrimal flow asymmetry after mood manipulation. We show that this is not reflected in LDS anatomy. Moreover, our detailed morphometric data can aid in therapeutic planning of LDS luminal procedures, especially when DCG images of one LDS are used as a roadmap for contralateral interventions. (Folia Morphol 2013; 72, 2: 137-141)
\end{abstract}

Key words: nasolacrimal duct, anatomy, epiphora, symmetry

\section{INTRODUCTION}

Major organ systems of vertebrates are structurally and functionally distinct on the left and right sides despite orderly symmetries of the exterior [14]. Interestingly, asymmetry in lacrimal flow is known to occur in females more than males [5].
Consequently, as a result of this disparity in function we tested the hypothesis that the normal lacrimal drainage system (LDS) might show subtle left-right asymmetry in its anatomy and morphometry when imaged on digital subtracted dacryocystography (DS-DCG). 
A parallel second goal of this research was to use the data obtained, consisting of accurate multiple morphometric measures of the LDS derived from high-resolution imaging, to establish the first comprehensive dataset for sizes of the LDS applicable as a reference when planning therapeutic luminal procedures on the LDS (e.g. dacryocystoplasty, endoscopy, stent placement, etc.) [4].

Lastly, we wished to address the question of whether it would be possible, in the rare instances that it might arise, to rely entirely on the morphometrics of a normal LDS contralateral to a pathologically distorted LDS that requires surgery, to guide the size of instruments and devices to be used during interventions on the abnormal side. Such reliance would require the pre-supposition of a high degree of symmetry between right and left LDSs, and would play out in practice, for example, when DS-DCG images of one LDS might be used as a digital roadmap for contralateral LDS interventions. This same logic of using symmetry/asymmetry of body structures for simulation and planning of surgical procedures has been alluded to previously in the context of endovascular or extravascular repair procedures of arteries seemingly symmetrical within the body [15]. Knowledge of the presence of symmetric morphology of the LDS can provide ophthalmic surgeons and radiologists with better quantitative estimates, which may be used to measure the integrity of the LDS before and after surgical procedures.

\section{MATERIALS AND METHODS}

Procedures and imaging studies were performed during routine work-up of patients based on clinical indications; no prospective imaging of normal volunteers was performed in this study. The great majority of clinical indications for performing DS-DCG on patients were the presence of symptomatic epiphora. We retrospectively reviewed all DS-DCG studies performed over a 7-year period at our institution to assemble a cohort of non-consecutive studies with completely normal anatomy on DS-DCG imaging findings despite the patients' symptoms. Their symptoms were not attributable to structural abnormalities of the LDS, which is not an infrequent finding of DCG studies in clinical practice.

All procedures were performed by 4 radiologists using the same standard technique after informed consent was obtained [7]. After placing the patient in the supine position, topical anaesthesia eye drops
( $0.5 \%$ proparacaine hydrochloride) were administered. A 0.012" catheter was connected to a disposable syringe containing non-ionic contrast medium (iopamidol, $300 \mathrm{mg}$ iodine per $\mathrm{mL}$ ). Its end was introduced into the lower or upper lacrimal punctum and held in position using adhesive tape. The head of the patient was fixed and the correct position checked on fluoroscopy. One to $2 \mathrm{~mL}$ of contrast medium was gently administered whilst acquiring digital subtraction images (Siemens AXIOM Artis) at 3 frames per second, until contrast medium was seen to spill into the nasal cavity. Only frontal images were obtained on a routine basis. Importantly, we wished to establish normative dimensions of the LDS on routinely available frontal DS-DCG images, since these are precisely the studies performed by most radiologists upon clinical referral, and viewed by ophthalmologists prior to any surgical interventions. As such, additional manoeuvres, e.g. lateral imaging or variations in injection pressures and consequent variable distension of the LDS, were not pertinent factors in our study.

According to the guidelines of the revised Declaration of Helsinki, this study reported the retrospective analysis of anonymised data (images) obtained from 'routine sources' where consent of individual patients and ethical approval for research analysis was therefore not considered necessary $[10,13]$. We retrospectively reviewed 60 normal bilateral DS-DCGs performed on 30 patients. Using a GE healthcare Centricity ${ }^{\mathrm{TM}}$ PACS-IW review workstation, 2 radiologists retrospectively analysed together all anonymised digital images and used electronic callipers to measure 7 parameters: (1) the combined length of either the superior or inferior canaliculus plus common canaliculus, (2) the angle subtended by the common canaliculus and lateral wall of the lacrimal sac, (3) the length of the lacrimal sac, (4) the width of the lacrimal sac, (5) the length of the nasolacrimal duct (NLD), (6) the width of proximal NLD, and (7) the width of the distal NLD. We tested the effect of independent variables (age, gender, and side) on the dependent variables (length, width, or angle) using regression analysis in Microsoft ${ }^{\circledR}$ Excel. We used 1-way analyses of variance (ANOVA) as regression models when the explanatory variables were dummies or qualitative in nature (gender and side) and the regressands were quantitative (length, width, and angle). We used linear regression analyses when the regressor (age) was quantitative. Statistical significance was set at $p<0.05$. 


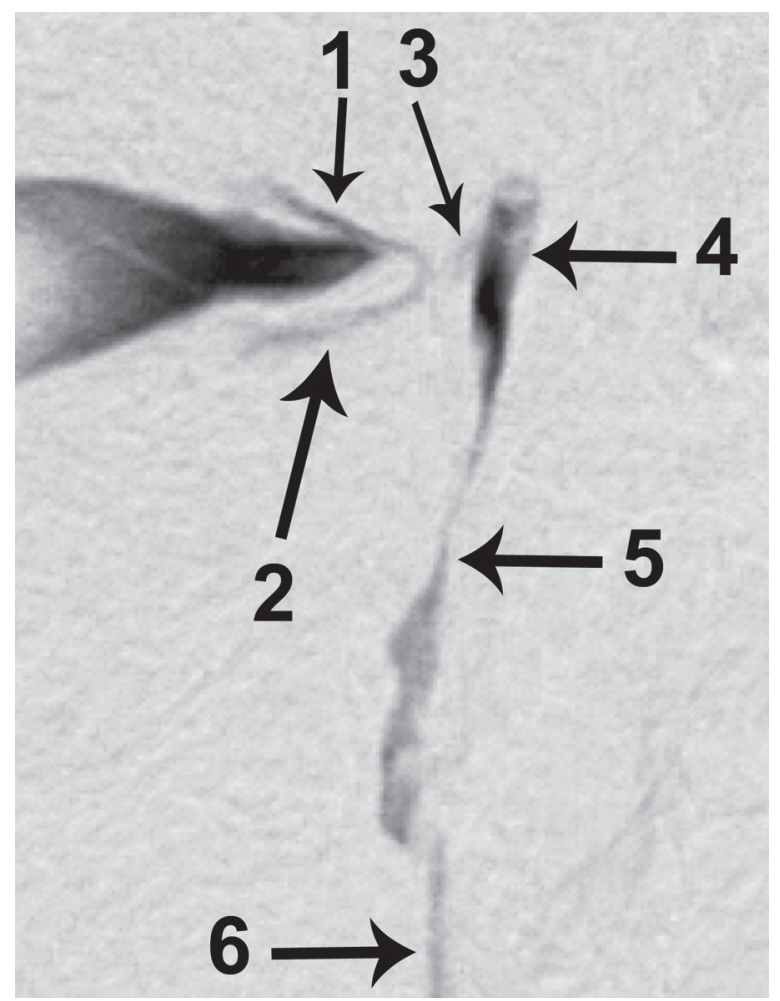

Figure 1. Right-sided digital subtracted dacryocystogram showing a normal lacrimal drainage system; 1 - superior canaliculus; 2 inferior canaliculus; 3 - common canaliculus; 4 - lacrimal sac; 5 - nasolacrimal duct; 6 - contrast medium in nasal cavity.

\section{RESULTS}

The patients ranged in age from 42 to 82 years (mean 62 years); $73 \%$ were female and $27 \%$ were male, which reflected the acknowledged more common occurrence of epiphora in females. All DS-DCGs were of good diagnostic quality, with sufficient pixel shifting that counteracted any motion artefacts, and which optimally outlined the course of the LDS free of surrounding bones (Fig. 1). The results are presented in detail as mean, standard deviation, and range, as displayed in Table 1. The results of regression analyses are presented in Table 2. None of the independent variables affected variations in length, size, and angles measured. No significant difference emerged between dimensions of the right and left LDSs.

\section{DISCUSSION}

Segmental canalisation of the ectodermal epithelial cords that develop into the LDS occurs simultaneously throughout their entire length between the fourth and eighth months of gestation [11]. The anatomy of the LDS has been described in detail

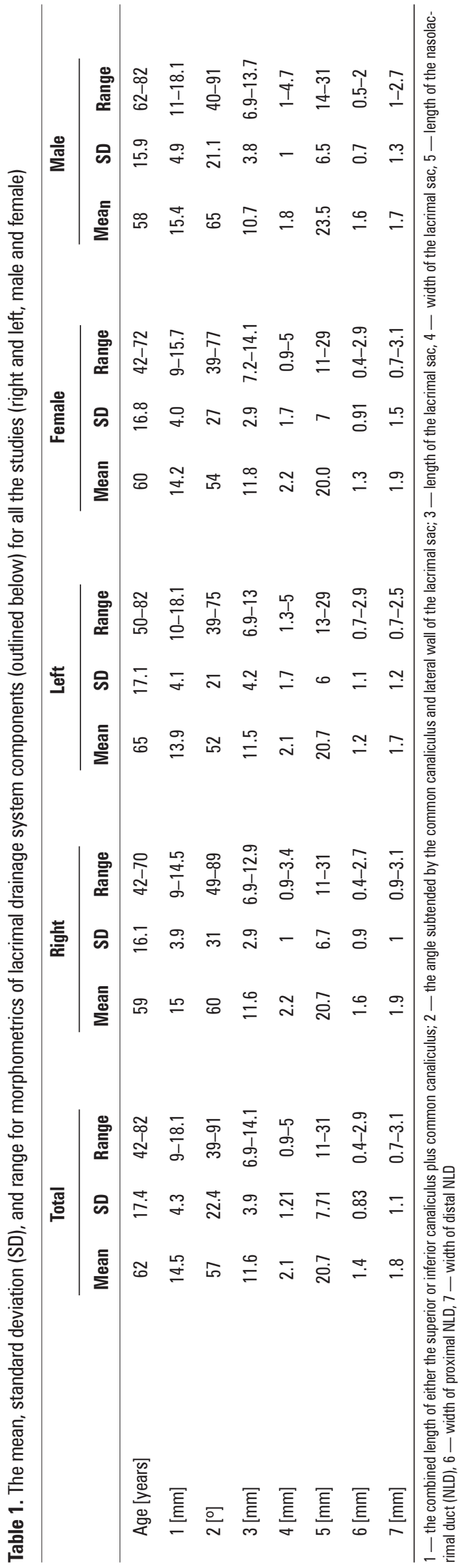


Table 2. The results of regression analyses: We tested the independent variables (age, gender, and side) against dependent variables (length, width, or angle, abbreviated as 1 to 7 , and outlined in detail below) using regression analysis. Significance set at $p<0.05$. $P$ values displayed. The coefficient of determination $\left(R^{2}\right)$ is also displayed for results of testing the effect of age on the dependent variables. None of the independent variables affected variation in length, width, or angle

\begin{tabular}{lcccccccc}
\hline & \multicolumn{7}{c}{ Dependent variables of the lacrimal drainage system (length, width, and angle, as outlined below) } \\
\cline { 2 - 6 } & $\mathbf{1}$ & $\mathbf{2}$ & $\mathbf{3}$ & $\mathbf{4}$ & $\mathbf{5}$ & $\mathbf{6}$ & $\mathbf{7}$ \\
\hline Age & 0.12 & 0.13 & 0.53 & 0.47 & 0.52 & 0.09 & 0.12 \\
& $\left(\mathrm{R}^{2}=0.10\right)$ & $\left(\mathrm{R}^{2}=0.09\right)$ & $\left(\mathrm{R}^{2}=0.2\right)$ & $\left(\mathrm{R}^{2}=0.09\right)$ & $\left(\mathrm{R}^{2}=0.04\right)$ & $\left(\mathrm{R}^{2}=0.11\right)$ & $\left(\mathrm{R}^{2}=0.13\right)$ \\
Gender & 0.58 & 0.23 & 0.81 & 0.58 & 0.71 & 0.89 & 0.47 \\
Side & 0.89 & 0.65 & 0.79 & 0.26 & 0.37 & 0.58 & 0.72 \\
\hline
\end{tabular}

1 - the combined length of either the superior or inferior canaliculus plus common canaliculus; 2 - the angle subtended by the common canaliculus and inferior wall of the lacrimal sac; 3 - length of the lacrimal sac; 4 - width of the lacrimal sac; 5 — length of the nasolacrimal duct (NLD); 6 - width of proximal NLD; 7 - width of distal NLD

Table 3. Representative morphometric dimensions of the lacrimal drainage system reported in the literature (references in brackets)

\begin{tabular}{lccccccc}
\hline & $\begin{array}{c}\text { This study } \\
\text { (mean) }\end{array}$ & $\begin{array}{c}\text { Burkat, } \\
\text { Lucarelli [4] }\end{array}$ & $\begin{array}{c}\text { Kassel, } \\
\text { Schatz [7] }\end{array}$ & $\begin{array}{c}\text { Malik } \\
\text { et al. [12] }\end{array}$ & $\begin{array}{c}\text { Kurihashi } \\
\text { et al. [9] }\end{array}$ & $\begin{array}{c}\text { Ashikaga } \\
\text { [2] }\end{array}$ & $\begin{array}{c}\text { Aoyama } \\
\text { et al. [1] }\end{array}$ \\
\hline Total length of canaliculi [mm] & 14.5 & $8-10$ & $10-17$ & - & 11.5 & 10.2 & - \\
Length of lacrimal sac [mm] & 11.6 & $12-15$ & - & 11.1 & 11.0 & 9.8 & - \\
Width of lacrimal sac [mm] & 2.1 & - & - & 2.4 & - & - & - \\
Length of nasolacrimal duct [mm] & 20.7 & - & - & 20.9 & 15.0 & 18.4 & 18.0 \\
Width of proximal nasolacrimal duct [mm] & 1.4 & - & - & 2.3 & - & - & - \\
Width of distal nasolacrimal duct [mm] & 1.8 & - & - & 2.3 & - & - & - \\
\hline
\end{tabular}

elsewhere [4]. In brief, tears from the conjunctival sac enter the puncta of both inferior and superior canaliculi. These 2 canaliculi merge to form the common canaliculus (common ampulla) and may enter directly, by 1 or 2 openings, or indirectly, by a dilated small diverticulum of the lateral wall of the lacrimal sac, into the sinus of Maier. The common canaliculus enters the lateral aspect of the nasolacrimal sac just above the junction of its upper and middle thirds. The nasolacrimal sac empties inferiorly into the NLD, which consists of a superior intraosseous portion and an inferior membranous portion. The intraosseous NLD travels posterolaterally through the nasolacrimal canal within the maxillary bone, while the membranous part runs within the nasal mucosa, eventually opening into the inferior meatus of the nose under the inferior turbinate. Representative but incomplete morphometric dimensions of the LDS reported in the literature are summarised in Table 3.

The first radiographic examinations of the LDS were carried out by Aubaret in 1911 and Szily in 1914 [8]. Since then, a variety of imaging techniques have been developed for the lacrimal apparatus, including DS-DCG, scintigraphy, ultrasonography, post-dacryocystographic computed tomography, and magnetic resonance dacryocystography [7]. The value of these modalities in the diagnosis of diseases of the LDS has been the subject of numerous studies. It is generally accepted that the modern imaging technique of DS-DCG is the gold standard for outlining the in vivo anatomy, luminal profile, and pathology of the LDS [6]. A typical DS-DCG image with a $1024 \times 1024$ matrix has an in-plane spatial resolution of $250 \mu \mathrm{m}$ [3]. Visualisation by DS-DCG reveals considerable variations in the structure of the sac and NLD among normal individuals. Nonetheless, in the normal DS-DCG, the canaliculi, lacrimal sac, and NLD are not usually dilated, and on the frontal images the lacrimal sac and NLD may have almost a linear configuration. In a patent LDS, there should be free flow of contrast through the NLD into the inferior meatus of the nasal cavity. Despite the non-distended state of a normal LDS, the high spatial resolution of the images is usually adequate to allow depiction of fine irregularities 
in luminal contour. This is optimal for the purposes of our study to obtain accurate and clinically relevant morphometrics and to depict the presence of any subtle left-right asymmetry in the anatomy of the LDS.

Major organ systems of vertebrates are structurally and functionally distinct on the left and right sides despite orderly symmetries of the exterior [14]. Interior asymmetries of each organ system appear to follow a concordant plan: their left-right orientation is not normally random and is consistent among distinct classes of vertebrates, suggesting an evolutionarily shared developmental mechanism [14]. It is believed that in the central nervous system (CNS), asymmetries in late embryological development foster the development of complex behavioural functions. Indeed, the role of CNS asymmetries in behavioural functions is currently an active area of study in developmental biology, including the question of the genetic control of asymmetry, ranging from behaviours based in brain functional asymmetry to simple morphological asymmetries [14].

A degree of affective lateralisation in the brain is known to result in lacrimal flow asymmetry after mood manipulation [5]. The functional and neuroanatomical patterning that may control this asymmetric lacrimal flow above the level of the facial nerve nucleus is unknown [5]. We show that this asymmetry is not substantiated in consequent anatomic and morphometric features of the LDS. Although the anatomy of the lacrimal glands themselves was not examined (any asymmetry of which may or may not contribute to explaining the known disparity of lacrimal flow), it would seem that further basic understanding of genetic and higher CNS control of lacrimal flow might depend on functional characteristics rather than reliance on evidence of morphological asymmetry of the LDS. Nonetheless, our findings of bilateral LDS symmetry, and the first comprehensive morphometric measurements we provide for the entire LDS, can be of practical use clinically in therapeutic planning for patients undergoing luminal procedures on the LDS (dacryocystoplasty, endoscopy, stent placement, etc.) [4] especially when DCG images of one LDS may be used as an aid and digital roadmap for contralateral LDS interventions.

\section{REFERENCES}

1. Aoyama M, Aikawa H, Yasutake K (1993) On the shape of the human lacrimal sac and the nasolacrimal duct. No. 1. Experimental study of Japense adult male cadavers. Acta Soc Opthalmol Jpa, 37: 1323-1331.

2. Ashikaga R (1925) Anatomy of the lacrimal drainage pathway. Acta Soc Opthalmol Jpn, 29: 67-128.

3. Bradley WG Jr. (2008) Pros and cons of 3 tesla MRI. J Am Coll Radiol, 5: 871-878.

4. Burkat CN, Lucarelli MJ (2006) The anatomy of the lacrimal system. In: Cohen AJ, Mercandetti M, Brazzo BG eds. The lacrimal system: diagnosis, management, and surgery. $1^{\text {st }}$ Ed. Springer, New York, pp. 3-20.

5. Delp MJ, Sackeim HA (1987) Effects of mood on lacrimal flow: Sex differences and asymmetry. Psychophysiology, 24: 550-556.

6. Francisco FC, Carvalho AC, Francisco VF, Neto GT (2007) Evaluation of 1000 lacrimal ducts by dacryocystography. Br J Opthalmol, 91: 43-46.

7. Kassel EE, Schatz CJ (1996) Lacrimal apparatus. In: Som PM, Curtin HD eds. Head and neck imaging. Mosby - Year Book, St. Louis, pp. 1129-1183.

8. Kirchhof K, Hähnel S, Jansen O, Zake S, Sartor K (2000) Gadolinium-enhanced magnetic resonance dacryocystography in patients with epiphora. J Comput Assist Tomogr, 24: 327-331.

9. Kurihashi K, Imada M, Yamashita A (1991) Anatomical analysis of the human lacrimal drainage pathway under an operating microscope. Int Ophthalmol, 15: 411-416.

10. Lawlor DA, Stone T (2001) Public health and data protection: an inevitable collision or potential for a meeting of minds? Int J Epidemiol, 30: 1221-1225.

11. Mafee MF (2003) Orbit: embryology, anatomy, and pathology. In: Som PM, Curtin HD eds. Head and Neck Imaging. $4^{\text {th }}$ Ed. Mosby, St. Louis, pp. 529-654.

12. Malik SR, Gupta AK, Chaterjee S, Bhardwaj OP, Saha M (1969) Dacryocystography of normal and pathological lacrimal passages. Br J Ophthalmol, 53: 174-179.

13. Schroter S, Plowman R, Hutchings A, Gonzales A (2006) Reporting ethics committee approval and patient consent by study design in five general medical journals. J Med Ethics, 32: 718-723.

14. Yost $\mathrm{HJ}$ (2009) Coordinating the development of bilateral symmetry and left-right asymmetry. Semin Cell Dev Biol, 20: 455.

15. Zurada A, Gielecki JS (2007) A novel formula for the classification of blood vessels according to symmetry, asymmetry and hypoplasia. Folia Morphol, 66: 339-345. 